\title{
A NOTE ON PARACOMPACTNESS AND NORMALITY OF MAPPING SPACES ${ }^{1}$
}

\author{
A. H. STONE
}

In a discussion with the author, J. C. Moore asked whether the space $Y^{\boldsymbol{X}}$ of mappings of a compact metric space $X$ in a locally compact paracompact space $Y$ is necessarily paracompact. (Throughout, "space" means "Hausdorff space," all mappings are understood to be continuous, and $Y^{\boldsymbol{x}}$ is given the compact-open topology.) It has been shown by Fadell [1] that if we drop the assumption that $Y$ is locally compact the answer is negative. We shall give two simple examples to show that not even the assumption that $Y$ is compact is sufficient. In both examples, as in Fadell's, $X$ will be the unit interval $I$, so that $Y^{\boldsymbol{X}}$ becomes the space of paths on $X$. In our first example, $Y$ is 1-dimensional (in the covering sense); in our second, $Y$ has infinite dimension but $Y^{x}$ is not even normal. In Fadell's example, $Y$ (though not compact) is 1-dimensional and $Y^{x}$ is also not normal. It would be interesting to know whether $Y^{I}$ is normal whenever $Y$ is both compact and finite-dimensional.

Let $L$ be the transfinite line from 0 to $\omega_{1}$ inclusive; this is the compact, totally ordered 1-dimensional space obtained by joining each countable ordinal to its successor by an interval, and adjoining $\omega_{1}$ (see, for example, [2, p. 263] for a formal definition of $L-\left(\omega_{1}\right)$ ).

\section{TheOREM 1. $L^{I}$ is not paracompact.}

Let $M$ be the subset of $L^{I}$ consisting of those maps $f: I \rightarrow L$ for which $f(0)=0$. Clearly $M$ is closed in $L^{I}$, and it will suffice to prove $M$ not paracompact. In what follows, $\alpha$ will run over the ordinals $<\omega_{1}$. Put $U_{\alpha}=\{f \mid f \in M$ and, for all $t \in I, f(t)<\alpha\}$; the sets $U_{\alpha}$ form an open covering of $M$. Let $V$ be any (open) refinement of $\left\{U_{\alpha}\right\}$; we show that $V$ is not locally finite.

We define a sequence of maps $f_{n} \in M$ and of countable ordinals $\alpha(n)(n=1,2, \cdots)$ as follows. Take $f_{1} \in M$ such that $f_{1}(1)>1$. Then $f_{1} \in$ some $V_{1} \in V$, and $V_{1} \subset$ some $U_{\alpha(1)}$. When $f_{n}$ and $\alpha(n)$ have been defined, take $f_{n+1} \in M$ such that (i) $f_{n+1}(1)>\alpha(n)$, (ii) $f_{n+1}(t) \geqq f_{n}(t)$ for all $t \in I$, and (iii) $f_{n+1}(t)=f_{n}(t)$ whenever $0 \leqq t \leqq 1-n^{-1}$. This is possible, because the subspace of $L$ consisting of points $\leqq \alpha(n)+1$ is order-homeomorphic to $I$. Then take $V_{n+1} \in \mathcal{V}$ containing $f_{n+1}$, and

Received by the editors June 5, 1961 and in revised form, November 3, 1961.

1 This work was supported in part by the Office of Naval Research under Contract No. 266(57) at Columbia University. 
choose $\alpha(n+1)$ so that $V_{n+1} \subset U_{\alpha(n+1)}$. Clearly $\alpha(1)<\alpha(2)<\cdots$; put $\sup \alpha(n)=\beta\left(<\omega_{1}\right)$. It is easy to see that, for each $t \in I, \lim _{n \rightarrow \infty} f_{n}(t)$ exists; call it $g(t)$. Thus $g(0)=0$ and $g(1)=\beta$. Further, $g$ is continuous, because (a) if $0 \leqq t<1$ there is a neighborhood of $t$ on which $f_{n}$ is ultimately constant, from (iii), and (b) if $t=1$ and $m$ is a given positive integer, we have $f_{m+1}(s)>\alpha(m)$ whenever $s$ is close enough to 1 , and then $\alpha(n)>f_{n}(s)>\alpha(m)$ for all $n>m$, proving $\beta \geqq g(s) \geqq \alpha(m)$. By Dini's theorem [3, p. 239] $f_{n} \rightarrow g$ uniformly, and hence $f_{n} \rightarrow g$ in $M$. Thus every neighborhood of $g$ meets $V_{n}$ for infinitely many values of $n$. But the sets $V_{n}$ are all distinct, since if $k>n$ we have $V_{n} \subset U_{\alpha(n)}$ and $f_{k} \in V_{k}-U_{\alpha(n)}$, by (i). Hence $V$ is not locally finite, and $M$ is not paracompact.

I do not know whether $L^{I}$ is normal. However, a compact (but infinite-dimensional) space $Y$ for which $Y^{I}$ is not normal can be obtained from the following lemmas:

(1) For any space $X$ and family of spaces $Y_{\lambda},\left(\prod Y_{\lambda}\right)^{\mathbf{x}}$ and $\Pi\left(Y_{\lambda}^{X}\right)$ are homeomorphic.

This is well known, and the proof is straightforward.

(2) If a product of nonempty spaces is normal, all but at most countably many of the factor spaces are countably compact.

This is [4, Theorem 3, Corollary].

(3) $I^{I}$ is not countably compact.

Again this is known; the proof consists in observing that the collection of maps $f_{n}(n=1,2, \cdots)$, where $f_{n}(t)=t^{n}$, has no cluster point (for the pointwise limit is discontinuous).

TheOREM 2. Let $Y$ be the product of uncountably many copies of the unit interval. Then $Y^{I}$ is not normal.

For, by (1), $Y^{I}$ is homeomorphic to $\Pi\left(I_{\lambda}^{I}\right)$, which by (2) and (3) is not normal.

The construction employed here raises the question: Under what conditions will $Y^{\boldsymbol{x}}$ be compact? The following partial answers are easily obtained.

THEOREM 3. A necessary and sufficient condition that $Y^{I}$ be compact is that $Y$ is compact and contains no arc.

We may identify $Y$ with a closed subset of $Y^{I}$, namely the set of constant functions; hence, if $Y^{I}$ is compact, so is $Y$. If $Y$ contains an arc $J$, then $J^{I}$ is a closed subset of $Y^{I}$, and (3) above shows that $Y^{I}$ cannot be compact. Conversely if $Y$ contains no arc, then each $f \in Y^{I}$ must be constant; for $f(I)$ is a Peano space (it is metrizable because, $Y$ being Hausdorff and $I$ compact, $f$ is closed) and so contains an 
arc if it has more than one point. Thus $Y^{I}$ and $Y$ are homeomorphic, which proves the sufficiency.

It is easily seen that in Theorem 3 we may replace "compact" by "countably compact" throughout, and $I$ (in either version) by any normal arcwise connected space of more than one point. Also the condition remains necessary if $I$ is replaced by any connected completely regular space of more than one point.

Finally, a similar argument but with $I$ replaced by the space $K$ formed by a single convergent sequence (plus its limit point) proves that the only spaces $Y$ for which $Y^{X}$ is compact for all compact metric spaces $X$ (or for $X=K$ ) are the spaces with at most one point.

The author is indebted to the referee for several helpful suggestions and improvements.

\section{REFERENCES}

1. E. Fadell, $B$ paracompact does not imply $B^{I}$ paracompact, Proc. Amer. Math. Soc. 9 (1958), 839-840.

2. L. Gillman and M. Jerison, Rings of continuous functions, Van Nostrand, Princeton, 1960.

3. J. L. Kelley, General Topology, Van Nostrand, New York, 1955.

4. A. H. Stone, Paracompactness and product spaces, Bull. Amer. Math. Soc. 54 (1948), 977-982.

UNIVERSITY OF ROCHESTER 